PAPER

\section{Effects of energy chirp on bunch length measurement in linear accelerator beams}

To cite this article: L Sabato et al 2017 Meas. Sci. Technol. 28084002

View the article online for updates and enhancements.
Related content

Transverse emittance-preserving arc compressor for high-brightness electron beam-based light sources and colliders

S. Di Mitri and M. Cornacchia

Temporal profile measurement of an electron bunch with the two-cell rf deflecting cavity at Waseda University Kazuyuki Sakaue, Yuichi Nishimura, Masahiro Nishiyama et al.

- Operating synchrotron light sources with a high gain free electron laser S Di Mitri and M Cornacchia

\section{Recent citations}

$$
\begin{aligned}
& \text { Effects of correlations between particle } \\
& \text { longitudinal positions and transverse plane } \\
& \text { on bunch length measurement: a case } \\
& \text { study on GBS electron LINAC at ELI-NP } \\
& \text { L Sabato et al }
\end{aligned}
$$




\title{
Effects of energy chirp on bunch length measurement in linear accelerator beams
}

\author{
L Sabato ${ }^{1}$, P Arpaia ${ }^{2}$, A Giribono ${ }^{3}$, A Liccardo ${ }^{2}$, A Mostacci ${ }^{3}$, L Palumbo ${ }^{3}$, \\ C Vaccarezza ${ }^{4}$ and A Variola ${ }^{4}$ \\ ${ }^{1}$ Dept. of Engineering, University of Sannio, Corso G. Garibaldi, 107, Benevento, Italy \\ 2 Dept. of Electrical Engineering and Information Technology, University of Naples Federico II, \\ Via Claudio 21, Naples, Italy \\ ${ }^{3}$ Dept. Scienze di Base e Applicate per l'Ingegneria (SBAI), University of Rome Sapienza, Via Antonio \\ Scarpa, 14, Rome, Italy \\ ${ }^{4}$ Istituto Nazionale di Fisica Nucleare-Laboratori Nazionali di Frascati (INFN-LNF), Via Enrico Fermi, \\ 40, Frascati, Italy \\ E-mail: luca.sabato@unisannio.it
}

Received 5 November 2016, revised 4 April 2017

Accepted for publication 11 April 2017

Published 17 July 2017

\begin{abstract}
The effects of assumptions about bunch properties on the accuracy of the measurement method of the bunch length based on radio frequency deflectors (RFDs) in electron linear accelerators (LINACs) are investigated. In particular, when the electron bunch at the RFD has a non-negligible energy chirp (i.e. a correlation between the longitudinal positions and energies of the particle), the measurement is affected by a deterministic intrinsic error, which is directly related to the RFD phase offset. A case study on this effect in the electron LINAC of a gamma beam source at the Extreme Light Infrastructure-Nuclear Physics (ELI-NP) is reported. The relative error is estimated by using an electron generation and tracking (ELEGANT) code to define the reference measurements of the bunch length. The relative error is proved to increase linearly with the RFD phase offset. In particular, for an offset of $7^{\circ}$, corresponding to a vertical centroid offset at a screen of about $1 \mathrm{~mm}$, the relative error is $4.5 \%$.
\end{abstract}

Keywords: electron bunch length measurement, LINAC, RF deflector, energy chirp, ELI-NP

(Some figures may appear in colour only in the online journal)

\section{Introduction}

Effective diagnostics are mandatory for achieving high brightness in high-repetition-rate machines. As an example, the gamma beam source (GBS) electron linear accelerator (LINAC) [1] can run at a maximum repetition rate of 100 $\mathrm{Hz}$ [2]. Therefore, at room temperature, the specifications of the requested spectral density $\left(10^{4}\right.$ photon $\left.(\mathrm{eV} \cdot \mathrm{s})^{-1}\right)$ cannot be achieved with single bunch collisions [3]. Multiple bunch collisions, with trains of 32 electron bunches separated by $16 \mathrm{~ns}$, distributed along a $0.5 \mu \mathrm{s}$ RF pulse, are needed. The bunches in each train have to be equally interspersed in order to recirculate the laser pulse in a suitable device, so as to allow the pulse to collide with all the electron bunches in the RF pulse before being dumped [3]. Moreover, the electron bunch length must be tuned according the laser bunch length.
Therefore, an important diagnostic task for the beam quality is to be able to measure the properties of a single bunch and a whole train of bunches [4].

In high-brightness electron LINACs, bunch length measurements are carried out mainly by means of a disruptive optical method, based on a radio frequency deflector (RFD), sometimes also called a transverse deflecting structure (TDS) $[5,6]$. The RFD provides a vertical kick to the electrons, introducing a correlation between the longitudinal and the vertical coordinates of the bunch. This allows the bunch length to be assessed through the vertical spot size measurement on a screen placed after the RFD. This method is based on an old idea [7], revamped after about 34 years [8]. The length of ultra-short electron beams can be measured by means of this method down to $50 \mathrm{fs}$ (beyond the range of streak cameras [9-11]). 
The method is widely used in high-brightness LINACs around the world. At the Stanford Linear Accelerator Center (SLAC) free electron laser [12], the use of multiple screens at appropriate advance phase locations has been proposed for monitoring the slice (i.e. time-correlated) transverse emittance [13]. A screen placed at a point of significant momentum dispersion has also been proposed to reveal how longitudinal phase space is populated [14]. At DESY, for the upcoming vacuum ultraviolet (VUV) free-electron laser (FEL) [15], for diagnosing very short bunches down to $50 \mathrm{fs,}$ a transverse deflecting RF structure (LOLA) was installed by achieving resolution well below 50 fs. At the Massachusetts Institute of Technology (MIT) Plasma Science and Fusion Center (PSFC), a fast diagnostic for the direct measurement of short bunch longitudinal phase space, with a time resolution of less than $100 \mathrm{fs}$, has been installed on the $17 \mathrm{GHz}$ LINAC. The two-fold aims of this were to measure very short electron bunches accurately, and also to provide online guidance for optimizing the injector and LINAC bunch length controls. These characteristics are needed for the operation of a chopper-prebuncher high-voltage injection system and a high-gradient $17 \mathrm{GHz}$ LINAC with an inherent ability to generate 1 degree (162 fs) electron bunches $[11,16]$. At the Sources for Plasma Accelerators and Radiation Compton with Lasers and Beams (SPARC-LAB) LINAC, the combination of an RFD and a dispersive system was shown capable of completely characterizing a 6D-beam phase space at the exit of a photoinjector [17]. However, the metrological performance of the method seems not to have been investigated yet [18]. Nevertheless, uncertainty surrounding the bunch length could mask significant pulse-to-pulse variations of electron beam parameters, generating undesired propagation on the beam quality as a whole.

In this paper, the measurement quality of the bunch length by RFD is shown to be affected by inaccuracy arising from some assumptions on the bunch properties at the RFD. In particular, when the bunch has a non-negligible energy chirp (i.e. the correlation between particle longitudinal positions and energies) at the RFD [19], the length measurement is affected by a deterministic intrinsic error, which is directly related to the RFD phase offset. In sections 2 and 3, the measurement technique and steps for the production of the model of measurement are detailed, respectively. In section 4 , the contribution of energy chirp to the bunch length measurement is analytically calculated. Finally, in section 5, a case study on the effects of the energy chirp on the bunch length measurements of the electron LINAC of the GBS at the Extreme Light Infrastructure-Nuclear Physics (ELI-NP) are evaluated in terms of relative error.

\section{Bunch length measurements by radio frequency deflector}

In this section, the measurement technique is explained in detail. First of all, the basic idea is introduced; secondly, the theory behind the measurement method is treated; finally, the measurement procedure steps are explained.
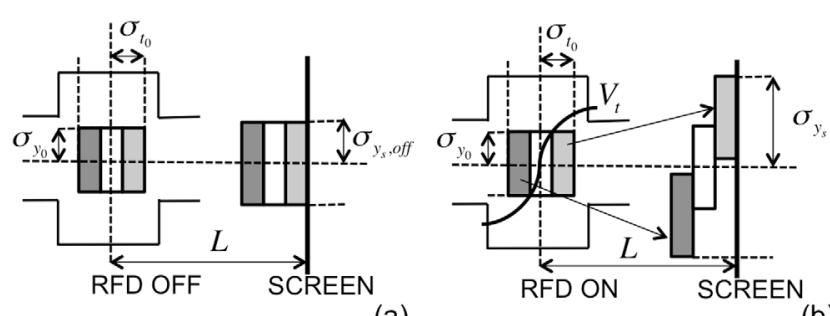

(a)

Figure 1. The behaviour of the longitudinal slices of the electron bunch after the RFD with (a) RFD off and (b) RFD on. The bunch is travelling from left to right. $\sigma_{y_{0}}$ is the vertical dimension of the bunch at the RFD centre, $L$ is the distance between the RFD centre and the screen, $V_{t}$ is the deflecting voltage amplitude, $\sigma_{t_{0}}$ is the bunch length, and $\sigma_{y_{s}, \text { off }}$ and $\sigma_{y_{s}}$ are the vertical spot sizes at the screen when the RFD is off and on, respectively.

\subsection{Basic idea}

When turned on, the RFD voltage introduces a correlation between the longitudinal coordinate of the particle and the vertical coordinate at the screen position (figure 1). In particular, a deflecting voltage phase is chosen in order to have a zero crossing of the transverse voltage in the centre of the bunch, giving a linear transverse deflection from head to tail. Consequently, the transverse displacement of each longitudinal bunch slice is proportional to its position with regard to the bunch centre [20] (figure 1). Therefore, information on the bunch length can be derived from the spot size measurements at the screen [17]. The example of the SPARC-LAB RFD cavity with specifications about the cavity and RF information is reported in [17].

\subsection{Theory}

The theory underlying the measurement method is based on an evaluation of the vertical bunch properties at the screen from the vertical and longitudinal bunch properties in both cases: (i) RFD off and (ii) RFD on.

(i) RFD off (figure 1(a)): in this case, there is a simple drift between the RFD and the screen. Thus, only the vertical position of the particle changes, while the vertical divergence does not vary. In particular, a particle, starting at the RFD with a vertical position of $y_{0}$ and a vertical divergence $y_{0}^{\prime}$, arrives at the screen with a vertical position $y_{s, \text { off }}$ and divergence $y_{s, \text { off }}^{\prime}[21]$ :

$$
\begin{gathered}
y_{s, \text { off }}=L y_{0}^{\prime}+y_{0}, \\
y_{s, \text { off }}^{\prime}=y_{0}^{\prime},
\end{gathered}
$$

where $L$ is the distance between the RFD centre and the screen. Equations (1) and (2) describe the vertical motion of the particle from the RFD to the screen with the RFD off. A particle bunch is described by its first- and secondorder statistical momenta (i.e. average and variance). The vertical spot size (i.e. the vertical dimension of the bunch) at the screen is:

$$
\sigma_{y_{s}, \text { off }}^{2}=\sigma_{y_{0}}^{2}+2 L \sigma_{y_{0} y_{0}^{\prime}}+L^{2} \sigma_{y_{0}^{\prime}}^{2}
$$


where $\sigma_{y_{0} y_{0}^{\prime}}$ is the covariance between the vertical position of the particle $y_{0}$ and divergence $y_{0}^{\prime}$ at the RFD centre, $\sigma_{y_{0}}$ and $\sigma_{y_{0}^{\prime}}$ are the variances of particle position and divergence at the RFD centre, respectively.

(ii) RFD on (figure 1(b)): each particle experiences a deflecting voltage when passing through the RFD, with the effect of changing its vertical divergence [21, 22]. The RFD can be modelled as (i) a drift of length $L_{\mathrm{RFD}} / 2$, (ii) a vertical kicker and (iii) another $L_{\mathrm{RFD}} / 2$ long drift, where $L_{\mathrm{RFD}}$ is the mechanical length of the RFD. Assuming that the bunch length is much smaller than the RF wavelength (i.e. $k z_{0} \ll 1$ ), the RFD voltage is $[12,14]$ :

$$
V\left(z_{0}\right) \approx V_{t}\left[k z_{0} \cos (\varphi)+\sin (\varphi)\right],
$$

where $z_{0}$ is the position of the particles along the beam axis with their origin in the RFD, and $k=2 \pi / \lambda_{\mathrm{RF}}, \lambda_{\mathrm{RF}}$, $V_{t}$ and $\varphi$ are the deflecting voltage wavelength, amplitude and phase, respectively. Therefore, the RFD gives a vertical divergence change:

$$
\Delta y_{0}^{\prime}\left(z_{0}\right)=C_{\mathrm{RFD}}\left[k z_{0} \cos (\varphi)+\sin (\varphi)\right],
$$

where $C_{\mathrm{RFD}}=V_{t} / E$ (in the ultra-relativistic regime) in which $E$ is the electron energy. Therefore, a particle arrives at the screen with the vertical position $y_{s}$ and divergence $y_{s}^{\prime}$ :

$$
\begin{gathered}
y_{s}=y_{0}+L\left(y_{0}^{\prime}+\Delta y_{0}^{\prime}\right), \\
y_{s}^{\prime}=y_{0}^{\prime}+\Delta y_{0}^{\prime},
\end{gathered}
$$

Equations (6) and (7) describe the vertical motion of the particle from the RFD to the screen with the RFD on. Assuming that (i) the averages of the vertical positions, divergences and longitudinal positions of the particle are null $\left(C_{y_{0}}=0 \mathrm{~m}, C_{y_{0}^{\prime}}=0 \mathrm{rad}\right.$, and $\left.C_{z_{0}}=0 \mathrm{~m}\right)$, and (ii) the energy chirp, the energy spread and the bunch correlations between the vertical and longitudinal planes are negligible, the vertical bunch centroid at the screen is $[12,14]$ :

$$
C_{y_{s}}=L C_{\mathrm{RFD}, 0} \sin (\varphi),
$$

where $C_{\mathrm{RFD}, 0}=V_{t} /\langle E\rangle$. The vertical spot size at the screen is $[12,14]:$

$$
\sigma_{y_{s}}^{2}=\sigma_{y_{s}, \text { off }}^{2}+K_{\mathrm{cal}}^{2} \sigma_{t_{0}}^{2},
$$

where $\sigma_{y_{s}}$,off is the vertical spot size at the screen with the RFD off (3), $\sigma_{t_{0}}$ is the bunch length (i.e. the longitudinal dimension of the bunch in seconds), and $K_{\mathrm{cal}}$ is the calibration factor:

$$
K_{\mathrm{cal}}=2 \pi f_{\mathrm{RF}} L C_{\mathrm{RFD}, 0} \cos (\varphi),
$$

where $f_{\mathrm{RF}}$ is the deflecting voltage frequency.

2.2.1. Calibration factor. The calibration factor is defined here as the coefficient relating the vertical coordinate at the screen to the longitudinal time coordinate of the bunch. By comparing (8) and (10), an important characteristic of this measurement method can be highlighted:

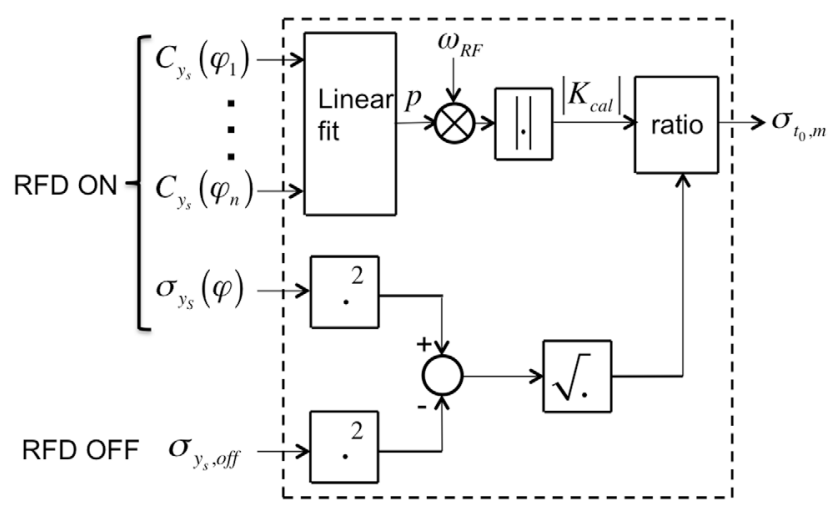

Figure 2. Model of the measurement production of the bunch length.

$$
K_{\mathrm{cal}}=2 \pi f_{\mathrm{RF}} \frac{\mathrm{d} C_{y_{s}}}{\mathrm{~d} \varphi} .
$$

Equation (11) shows that (i) the coefficient $K_{\text {cal }}$ can be directly calculated by measuring the centroid position of the bunch at the screen for different values of the RFD phase, and (ii) only the deflecting voltage angular frequency needs to be known, i.e. the measurements can be self-calibrated [20].

From (9), some further considerations arise regarding this measurement method: for the vertical spot size, only the second term contains information about the longitudinal bunch length (i.e. $\sigma_{t_{0}}$ ). Therefore, the second term should be non-negligible with respect to the first term. With this aim, (i) $K_{\text {cal }}$ can be increased (namely, the distance between the RFD and the screen and/or the deflecting voltage amplitude), and (ii) $\sigma_{y_{s} \text {,off }}$ can be decreased, by means of the vertical focusing quadrupoles placed before the RFD.

\section{Model of measurement production}

The model of the production of the bunch length measurement relies on the following operations (figure 2):

- with RFD OFF, measure $\sigma_{y_{s, \text { fff }}}$ - the vertical spot size at the screen;

- with RFD ON, measure $C_{y_{s}}\left(\varphi_{i}\right)$-the vertical bunch centroid at the screen for different values of the RFD phase $\varphi_{i}$;

- calculate the calibration factor $K_{\text {cal }}$, by multiplying the deflecting voltage angular frequency by the slope $p$ of the vertical bunch plot centroid at the screen versus $\varphi$, by means of a linear fit (11);

- measure $\sigma_{y_{s}}$-the vertical spot size at the screen with RFD $\mathrm{ON}$;

- measure the bunch length $\sigma_{t_{0}, m}$ by means of (9):

$$
\sigma_{t_{0}, m}=\frac{\sqrt{\sigma_{y_{s}}^{2}-\sigma_{y_{s}, \text { off }}^{2}}}{\left|K_{\mathrm{cal}}\right|}
$$

using the information from the previous steps.

With this measurement technique, the beam spot size at the screen with RFD off is designed to be very small by means of 
Table 1. RFD parameters.

\begin{tabular}{lll}
\hline$V_{t}(\mathrm{MV})$ & $f_{\mathrm{RF}}(\mathrm{GHz})$ & $L(\mathrm{~m})$ \\
\hline 1 & 2.856 & 1.1380
\end{tabular}

Table 2. The GBS electron LINAC bunch parameters of the vertical plane at the RFD entrance. $\sigma_{y}$ and $\sigma_{y^{\prime}}$ are expressed in rms.

\begin{tabular}{lllll}
\hline$C_{y}(\mathrm{~nm})$ & $C_{y^{\prime}}(\mathrm{nrad})$ & $\sigma_{y}(\mathrm{~mm})$ & $\sigma_{y^{\prime}}(\mu \mathrm{rad})$ & $\sigma_{y y^{\prime}}(\mathrm{mm} \cdot \mathrm{mrad})$ \\
\hline 0.655 & -3.36 & 0.354 & 57.6 & $-1.99 \cdot 10^{-2}$ \\
\hline
\end{tabular}

Table 3. The GBS electron LINAC bunch parameters of the longitudinal plane at the RFD entrance. $\sigma_{t_{0}}$ and $\sigma_{\delta}$ are expressed in rms.

\begin{tabular}{llll}
\hline$\langle E\rangle(\mathrm{MeV})$ & $\sigma_{t_{0}}(\mathrm{ps})$ & $\sigma_{\delta}$ & $\sigma_{t_{0} \delta}(\mathrm{fs})$ \\
\hline 118 & 0.912 & $6.05 \cdot 10^{-3}$ & 5.33 \\
\hline
\end{tabular}

the vertical focusing quadrupole in order to improve the resolution. For this reason, the jitter of the beam spot size at the screen with RFD off can be neglected. In a Compton machine (i.e. high-brightness electron LINAC), the jitter of the particle energy average is negligible.

\section{Accuracy loss due to energy chirp}

In this section, the effect of energy chirp on the bunch length measurement is analytically calculated. With this aim, the vertical centroid and the spot size at the screen are determined, using the same assumptions as those in section 2: (i) the averages of the vertical positions, divergences and longitudinal positions of the particle are null, and (ii) the energy spread and bunch correlations between the vertical and longitudinal planes are negligible, except for a non-negligible energy chirp. With these assumptions, the vertical centroid at the screen after the RFD is:

$$
C_{y_{s}}=L C_{\mathrm{RFD}, 0} \sin (\varphi)+L \frac{2 \pi f_{\mathrm{RF}}}{c}\left\langle C_{\mathrm{RFD}} z_{0}\right\rangle \cos (\varphi)
$$

Using the first-order Taylor series approximation of the inverse of particle energies around the energy average, the vertical centroid at the screen is:

$$
C_{y_{s}}=L C_{\mathrm{RFD}, 0} \sin (\varphi)-L C_{\mathrm{RFD}, 0} 2 \pi f_{\mathrm{RF}} \sigma_{t_{0} \delta} \cos (\varphi),
$$

where $\delta=(E-\langle E\rangle) /\langle E\rangle$, and $\sigma_{t_{0} \delta}$ is the energy chirp expressed in terms of covariance between the longitudinal positions and energies of the particle. A comparison of (8) and (14) highlights that the contribution of the energy chirp to the vertical centroid at the screen is expressed as the second term of (14). Considering small variations of the RFD phase around $0^{\circ}$ and $180^{\circ}$, the contribution of the energy chirp is a constant term which does not modify the slope of the straight line; therefore (11) can be used to calculate the calibration factor from the vertical bunch centroid measurements at the screen.

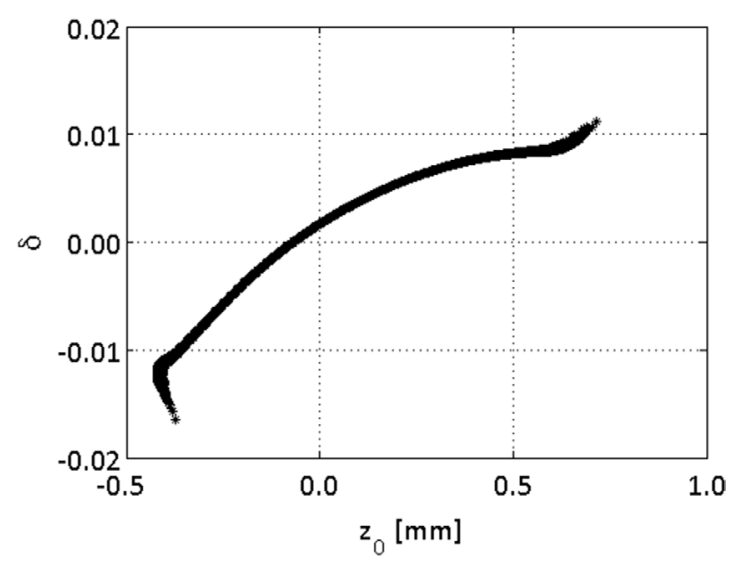

Figure 3. Longitudinal trace space of the bunch before RFD.

The vertical spot size at the screen is:

$$
\begin{aligned}
\sigma_{y_{s}}^{2}= & \sigma_{y_{s}, \text { off }}^{2}+K_{\mathrm{cal}}^{2} \sigma_{t_{0}}^{2}+L^{2} \frac{2 \pi f_{\mathrm{RF}}}{c} \sin (2 \varphi) \\
& \times\left[\left\langle C_{\mathrm{RFD}}^{2} z_{0}\right\rangle-C_{\mathrm{RFD}, 0}\left\langle C_{\mathrm{RFD}} z_{0}\right\rangle\right] .
\end{aligned}
$$

Using the first-order approximation of the Taylor series of the inverse of particle energies around the energy average, the vertical spot size at the screen is:

$$
\sigma_{y_{s}}^{2}=\sigma_{y_{s}, \text { off }}^{2}+K_{\mathrm{cal}}^{2} \sigma_{t_{0}}^{2}-L^{2} 2 \pi f_{\mathrm{RF}} C_{\mathrm{RFD}, 0}^{2} \sigma_{t_{0} \delta} \sin (2 \varphi) .
$$

Comparing (9) and (16), the contribution of the energy chirp to the vertical centroid at the screen can be recognized as the last term of (16). Choosing the deflecting voltage phase of $0^{\circ}$ and $180^{\circ}$ (in order to have a zero crossing of the transverse voltage at the centre of the bunch), this term is null. Thus, (9) predicts the vertical spot size at the screen well. When there is a deflecting voltage phase offset, the effect of energy chirp on the vertical spot size-given by the third term of (16)-becomes non-negligible, and (9) may not be able to predict the vertical spot size at the screen well. Therefore, the systematic error due to energy chirp can be mitigated by choosing an RFD phase which is as close as possible to $0^{\circ}$ or $180^{\circ}$.

Equations (14) and (16) are calculated in the more general case when the energy chirp of the electron bunch at the RFD entrance cannot be neglected. When the energy chirp is negligible, the (14) and (16) coincide with the equations of the vertical centroid (8) and the spot size at the screen (9) of section 2, respectively. For these reasons, the measurement procedure of the bunch length in section 3 could lead to a systematic error, when the bunch at the RFD is characterized by a non-negligible energy chirp. In particular, the bunch length measurement relative error is (using (12) with $\sigma_{y_{s}}$ given by (16)):

$$
\begin{aligned}
E_{r} & =\frac{\left|\sigma_{t_{0}}-\sigma_{t_{0}, m}\right|}{\sigma_{t_{0}}} \\
& =\frac{\left|\sigma_{t_{0}}-\sqrt{\sigma_{t_{0}}^{2}-2 \pi f_{\mathrm{RF}} \frac{L^{2}}{K_{\mathrm{cal}}^{2}} C_{\mathrm{RFD}, 0}^{2} \sigma_{t_{0} \delta} \sin (2 \varphi)}\right|}{\sigma_{t_{0}}},
\end{aligned}
$$




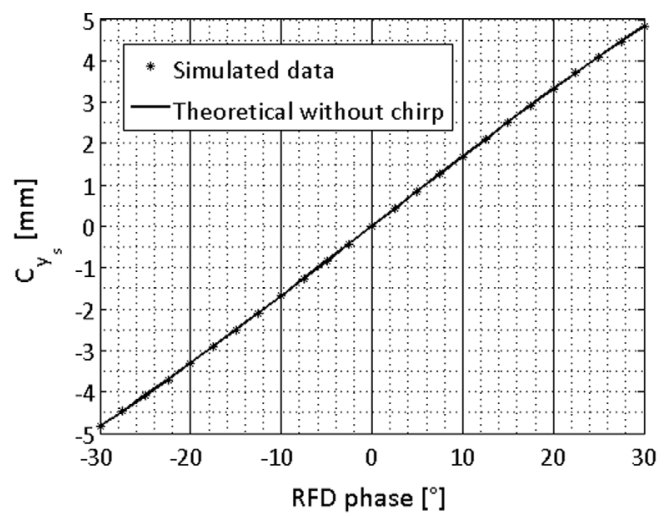

(a)

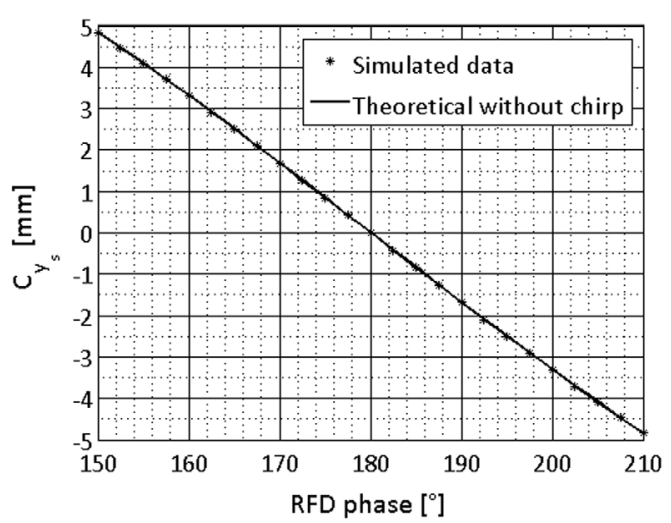

(b)

Figure 4. The vertical bunch centroid at the screen versus the RFD phase: the simulated data $\left({ }^{*}\right)$ and theoretical predictions without the contribution of an energy chirp (solid line) from (8) (using the values of the tables 1 and 3), choosing the RFD phase centre (a) $0^{\circ}$ and (b) $180^{\circ}$.

where $\sigma_{t_{0}}$ is the true bunch length and $\sigma_{t_{0}, m}$ is the measured bunch length by means of the model of the measurement production section 3 .

\section{Case study on the GBS electron LINAC}

In this section, the effects of the energy chirp on the bunch length measurements of the electron LINAC of the GBS at the ELI-NP are evaluated in terms of relative error.

ELI-NP is one of the pillars of the ELI European project dedicated to high-level research on ultra-high intensity lasers, laser-matter interaction and secondary sources [23]. The GBS at ELI-NP will be an advanced source of up to $20 \mathrm{MeV}$ gamma rays based on Compton back-scattering, i.e. the collision of an intense high-power laser beam and a high-brightness electron beam with a maximum kinetic energy of about $720 \mathrm{MeV}$ [3]. This infrastructure will be built in Magurele, near Bucharest (Romania). The purposes of this machine are various: to cover frontier fundamental physics, new nuclear physics and astrophysics as well as applications in material and life sciences, industrial tomography and nuclear waste management [23].

\subsection{Simulation conditions}

The bunch length measurements of the ELI-NP electron LINAC were simulated by using the electron generation and tracking (ELEGANT) code for tracking the particles from the RFD to the screen [24].

A bunch composed of 50000 particles was tracked by means of the ELEGANT code from the RFD LEL-RF-TDC01 to the screen LEL-DIA-SCN08 of the GBS electron LINAC, placed between the first and second C-band accelerating section [3]. The RFD parameters, as well as the bunch parameters of the vertical and longitudinal planes at the RFD entrance, are reported in tables $1-3$, respectively. The bunch travels parallel to the accelerator axis $\left(C_{y^{\prime}} \approx 0 \mathrm{rad}\right)$ and just on the axis itself $\left(C_{y} \approx 0 \mathrm{~m}\right)$. The energy chirp is not negligible $\left(\sigma_{t_{0} \delta}=5.33 \mathrm{fs}\right)$, as shown by the longitudinal trace space of the bunch before the RFD in figure 3. The RFD is modelled as a travelling wave cavity in ELEGANT (RFDF element).
This cavity provides a constant transverse deflection along the transverse coordinates. It is probably the best actual model, because real cavities contain a mixture of TM- and TE-like modes, resulting in uniform deflection [25].

\subsection{Numerical results}

In this section, the simulation results and theoretical predictions of the vertical centroid and spot size at the screen are compared at varying RFD phases centred on $0^{\circ}$. Finally, the relative error of the virtual bunch length measurement is calculated.

5.2.1. Vertical centroid. A satisfying agreement between the simulated (stars) and theoretical vertical bunch centroid at the screen versus the RFD phase without the contribution of energy chirp (solid line, from (8)) is highlighted in figure 4. In the case of the GBS electron LINAC, the contribution of the energy chirp to the vertical bunch centroid at the screen is negligible, because the second term of (14) is smaller than $1 \mu \mathrm{m}$ and the first term of (14) is on the order of mm (see figure 4). Moreover, the contribution of the energy spread to the vertical centroid at the screen is negligible, because the theoretical predictions without the energy spread contribution (8) show good agreement with the simulated data.

5.2.2. Vertical spot size. In figure 5, the simulated (stars) and the theoretical vertical spot size at the screen versus the RFD phase are reported, both with (solid line from (16)) and without (dashed, from (9)) the contribution of the energy chirp. The contribution of the energy chirp to the vertical spot size at the screen is non-negligible; therefore, the bunch length measurements are affected by a systematic error using the method illustrated in section 2. Conversely, the energy spread does not affect the vertical spot size at the screen. In figure 5, a dissymmetry in the vertical spot size at the screen with respect to $0^{\circ}$ (or $180^{\circ}$ ) due to the energy chirp is highlighted. A bunch with a non-negligible energy chirp means that the particle energy is related to the longitudinal position of the particle. Therefore, if the particles have more energy at the bunch head (or tail), 


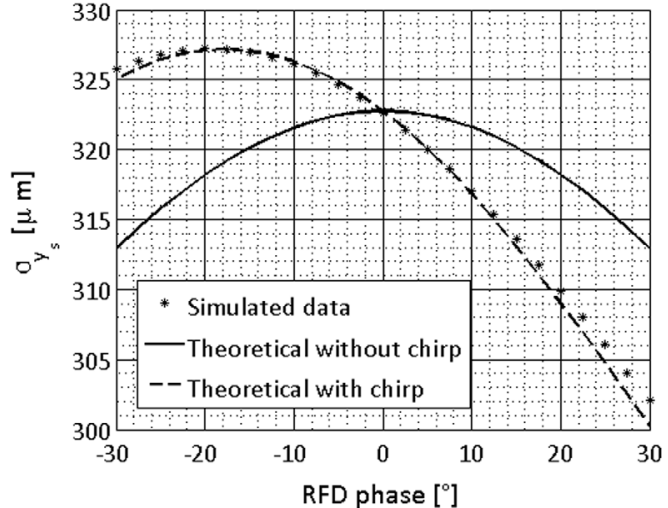

(a)

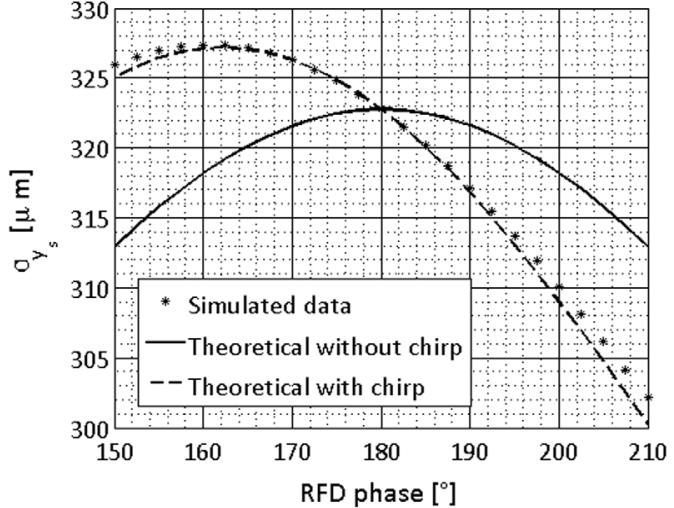

(b)

Figure 5. The vertical spot size at the screen versus the RFD phase: simulated data $\left({ }^{*}\right)$, theoretical predictions without the contribution of energy chirp (solid line) from (9), and theoretical predictions with the contribution of the energy chirp (dashed line) (16) (using values of tables 1-3), choosing the RFD phase centre (a) $0^{\circ}$ and (b) $180^{\circ}$.

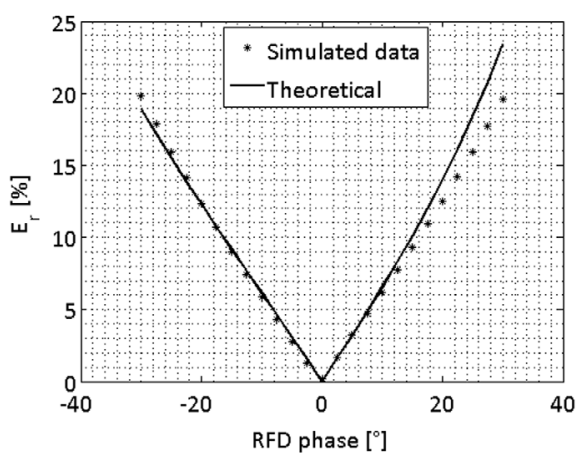

(a)

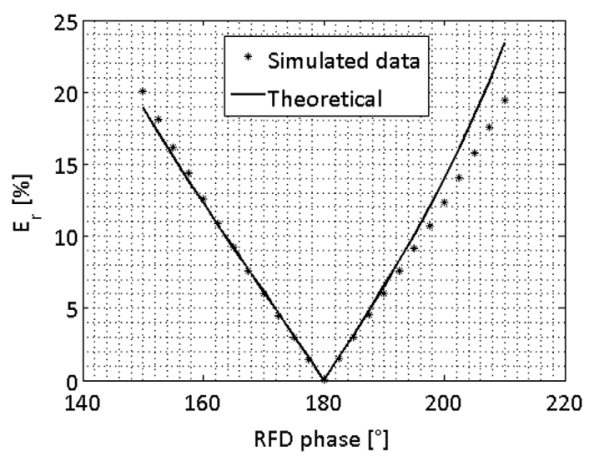

(b)

Figure 6. The relative error of the bunch length measurement versus the RFD phase: simulated data (stars) and theoretical predictions (solid line) of (17) (using the values of the tables 1-3), choosing the RFD phase centre (a) $0^{\circ}$ and (b) $180^{\circ}$.

the vertical spot size varies in a different way, choosing an RFD phase that is greater or less than $0^{\circ}\left(\right.$ or $\left.180^{\circ}\right)$.

\subsubsection{Relative error of the bunch length measurement. In} figure 6, the relative error of the bunch length measurement is plotted versus the RFD phase, for both the simulated data (stars) and theoretical predictions from (17) (solid line). The simulated relative error is given by $E_{r}=\left|\sigma_{t_{0}}-\sigma_{t_{0}, m}\right| / \sigma_{t_{0}}$, where $\sigma_{t_{0}}$ is the true bunch length and $\sigma_{t_{0}, m}$ is the measured bunch length obtained by means of the model of the measurement production of section 3 . The theoretical relative error only explains the contribution of the energy chirp using the model of the measurement production of section 3. Conversely, the simulated bunch length also takes into account other sources of systematic errors (e.g. energy spread, correlations between the vertical and longitudinal planes, and so on). A satisfying agreement between the theoretical predictions and simulated data for the RFD phase values near $\varphi=0^{\circ}$ (or $\varphi=180^{\circ}$ ) is highlighted. When $\varphi \neq 0^{\circ}$ (or $\varphi \neq 180^{\circ}$ ), the agreement worsens because the neglected terms become larger. The light dissymmetry of the theoretical relative error when the RFD phase is far from $0^{\circ}$ (or $180^{\circ}$ ) is due to the energy chirp, which is the source of the dissymmetry of the vertical spot size at the screen, and therefore also of the error dissymmetry. Furthermore, when the
RFD phase offset is equal to $0^{\circ}$ (or $180^{\circ}$ ), the relative error is minimum, because the energy chirp contribution becomes negligible (17). Conversely, when $\varphi \neq 0^{\circ}$ (or $\varphi \neq 180^{\circ}$ ), the relative error increases linearly according to the RFD phase offset. For an RFD phase offset of $7^{\circ}$, which corresponds to a vertical centroid offset at the screen on the order of $1 \mathrm{~mm}$ (figure 4), the relative error is on the order of $4.5 \%$.

\section{Conclusions}

The impact of an energy chirp on the bunch length measurement was assessed. The energy chirp was able to affect the vertical centroid at the screen, but considering the small variations of the RFD phase around $0^{\circ}$ and $180^{\circ}$, the contribution of the energy chirp is a constant term and does not modify the slope of the straight line. Therefore, the calibration factor can be calculated from the vertical bunch centroid measurements at the screen. Moreover, the energy chirp was able to affect the vertical spot size at the screen, and therefore the bunch length measurement procedure led to a measurement that had a systematic error. When the RFD phase offset is equal to $0^{\circ}$ (or $180^{\circ}$ ) the relative error is minimum, because the contribution of the energy chirp becomes negligible. The contribution of the energy chirp to the bunch length measurement increases according to the RFD phase offset (and therefore the vertical 
centroid offset at the screen). In conclusion, in the case of a bunch with a non-negligible energy chirp, the vertical centroid offset plays a fundamental role in the relative error of the bunch length measurement. Therefore, the systematic error due to the energy chirp can be mitigated by selecting an RFD phase that is as close as possible to $0^{\circ}$ or $180^{\circ}$.

In the case of the GBS electron LINAC at ELI-NP, the contribution of energy chirp to the vertical bunch centroid at the screen is negligible; therefore, the calibration factor can be calculated from vertical bunch centroid measurements. Conversely, the contribution of the energy chirp to the vertical spot size at the screen is non-negligible; therefore, the bunch length measurements are affected by a deterministic error. When the RFD phase offset is null, the relative error is minimum. Conversely, the relative error increases linearly with the RFD phase offset. In particular, for an offset of $7^{\circ}$, corresponding to a vertical centroid offset at the screen on the order of $1 \mathrm{~mm}$, the relative error of the measurement is on the order of $4.5 \%$.

Uncertainty sources due to other bunch parameters will be investigated. In particular, the correlations between the longitudinal and vertical plane (i.e. the vertical and longitudinal position of the particle, and the vertical divergence and longitudinal position of the particle) and the energy spread can affect the bunch length measurements using an RFD.

\section{Acknowledgments}

The authors would like to thank A Cianchi and M Borland for useful suggestions and discussions. The authors also thank the anonymous reviewers for their useful suggestions, which improved the content and readability of the paper significantly.

\section{References}

[1] Giribono A et al 2016 6D phase space electron beam analysis and machine sensitivity studies for ELI-NP GBS Nucl. Instrum. Methods Phys. Res. A 829 274-7

[2] Bacci A et al 2013 Electron LINAC design to drive bright Compton back-scattering gamma-ray sources J. Appl. Phys. 113194508

[3] Serafini L et al 2014 Technical design report EuroGammaS proposal for the ELI-NP gamma beam system (arXiv:1407.3669)

[4] Cianchi A et al 2015 Six-dimensional measurements of trains of high brightness electron bunches Phys. Rev. Spec. Top. Accel. Beams 18082804

[5] Cianchi A, Anania M P, Bisesto F, Castellano M, Chiadroni E, Pompili R and Shpakov V 2016 Observations and diagnostics in high brightness beams Nucl. Instrum. Methods Phys. Res. 829 343-7

[6] Ding Y, Behrens C, Emma P, Frisch J, Huang Z, Loos H, Krejcik P and Wang M H 2011 Femtosecond x-ray pulse temporal characterization in free-electron lasers using a transverse deflector Phys. Rev. Spec. Top. Accel. Beams 14120701

[7] Miller R H, Koontz R F and Tsang D D 1965 The SLAC injector IEEE Trans. Nucl. Sci. 3 804-8
[8] Wang X J 1999 Producing and measuring small electron bunches Proc. Particle Accelerator Conf. vol 1 (IEEE) pp 229-33

[9] Li B et al 2009 Design of a sub 100-femtosecond x-ray streak camera Conf. Lasers and Electro-Optics (Optical Society of America) pp 85-9

[10] Bentson L, Emma P and Krejcik P 2002 A new bunch compressor chicane for the SLAC LINAC to produce $30 \mathrm{fsec}, 30 \mathrm{ka}, 30 \mathrm{Gev}$ electron bunches EPAC-2002, Paris, France pp 682-5

[11] Haimson J, Mecklenburg B, Stowell G and Ishii B 2002 A circularly polarized beam deflector for direct measurement of ultra short electron bunches AIP Conf. Proc. vol 647 (AIP) pp 810-20

[12] Akre R, Bentson L, Emma P and Krejcik P 2001 A transverse $\mathrm{RF}$ deflecting structure for bunch length and phase space diagnostics Proc. 2001 Particle Accelerator Conf. vol 3 (IEEE) pp 2353-5

[13] Ben-Zvi I, Qiu J X and Wang X 1997 Picosecond-resolution slice emittance measurement of electron-bunches Proc. 1997 Particle Accelerator Conf. 1997 vol 2 pp (IEEE) $1971-5$

[14] Emma P, Frisch J and Krejcik P 2000 A transverse RF deflecting structure for bunch length and phase space diagnostics $L C L S$ Tech. Note 12 LCLS-TN-00-12 (www-ssrl.slac.stanford.edu/ lcls/technotes/LCLS-TN-00-12.pdf)

[15] Hüning M, Bolzmann A, Schlarb H, Frisch J, McCormick D, Ross M, Smith T and Rossbach J 2005 Observation of femtosecond bunch length using a transverse deflecting structure Proc. FEL (Stanford, California) (www.slac. stanford.edu/cgi-wrap/getdoc/slac-pub-11482.pdf)

[16] Haimson J 2004 Longitudinal phase space measurements of short electron bunches using a $17 \mathrm{GHz}$ circularly polarized beam deflector In AIP Conf. Proc. vol 737 (AIP) pp 95-108

[17] Alesini D, Di Pirro G, Ficcadenti L, Mostacci A, Palumbo L, Rosenzweig J and Vaccarezza C 2006 RF deflector design and measurements for the longitudinal and transverse phase space characterization at SPARC Nucl. Instrum. Methods Phys. Res. A 568 488-502

[18] Sabato L, Alesini D, Arpaia P, Giribono A, Liccardo A, Mostacci A, Palumbo L, Vaccarezza C and Variola A 2016 Metrological characterization of the bunch length system measurement of the ELI-NP electron LINAC 14TH IMEKO TC-10 (Milan 2016) pp 203-8

[19] Sabato L, Arpaia P, Liccardo A, Alesini D, Franzini G, Vaccarezza C, Variola A, Giribono A, Mostacci A and Palumbo L 2016 Longitudinal phase space measurement at the ELI-NP Compton gamma source Proc. International Beam Instrumentation Conference (September 11-15, Barcelona, Spain)

[20] Alesini D et al 2009 Sliced beam parameter measurements Proc. DIPAC (Basel, Switzerland, May 2009) TUOA-01

[21] Wiedemann H 2007 Particle Accelerator Physics (Berlin: Springer)

[22] Bongardt K 1981 Calculation of the transfer matrix $t$ in six dimensions for an RF-deflector element Technical Report Los Alamos Scientific Lab., NM (USA) (https://doi.org/10.2172/6683073)

[23] Ur C A, Balabanski D, Cata-Danil G, Gales S, Morjan I, Tesileanu O, Ursescu D, Ursu I and Zamfir N V 2015 The ELI-NP facility for nuclear physics Nucl. Instrum. Methods Phys. Res. B 355 198-202

[24] Borland M 2000 ELEGANT: a flexible SDDS-compliant code for accelerator simulation Technical Report Argonne National Lab., IL, USA

[25] Borland M 2017 Users manual for ELEGANT http://www. aps.anl.gov/Accelerator_Systems_Division/Accelerator_ Operations_Physics/manuals/elegant_latest/elegant.pdf 